serious threat worldwide. Gonorrhea is a sexually transmitted disease with a high morbidity burden and is an important cause of pelvic inflammatory disease. The failure of recommended dual therapy with ceftriaxone and azithromycin has compromised the general and reproductive health of infected individuals. Thereby, Neisseria gonorrhoeae was recently classified as a 'Priority 2' microorganism by the World Health Organization. Consequently, persistent attempts are under way to discover novel drug targets as well as new drugs to fight against Neisseria. In this direction, considerable number of phytochemicals have been reconnoitred for their remedial intercession via targeting bacterial proteins.

Methods MurI gene is specific to the bacterial kingdom, it can be exploited as a potential drug target for the treatment of bacterial diseases. Accordingly, diverse families of phytochemicals were screened in silico for their binding affinity with NG-MurI protein. Esculetin, one of the shortlisted compounds, was evaluated for its functional, structural and antibacterial activity. MurI was cloned, expressed and purified to homogeneity and used for testing the effect of esculetin on its racemase activity under invitro conditions. We further evaluated the effect of esculetin on sensitive and drug resistant strains of NG.

Results We screened various classes of natural compounds and found esculetin, a coumarin derivative as a potent compound to target its effect on the growth of Neisseria gonorrhoeae. Treatment with esculetin resulted in growth inhibition, cell wall damage and altered permeability as revealed by fluorescence and electron microscopy. Furthermore, esculetin inhibited racemization activity of recombinant, purified MurI protein of NG, an important enzyme required for peptidoglycan biosynthesis.

Conclusions Our results suggest that esculetin could be further explored as a lead compound for developing new drug molecules against multidrug resistant strains.

\section{P194 PARTICIPATORY COMMUNITY-BASED MAPPING OF PEOPLE WHO INJECT DRUGS (PWID) LOCATIONS TO IMPROVE HIV CASE-FINDING}

${ }^{1} \mathrm{O}$ Kovtun*, ${ }^{2} \mathrm{M}$ Malakhova, ${ }^{1} \mathrm{~T}$ Saliuk. ${ }^{1}$ The International Charitable Foundation 'Alliance for Public Health', Kyiv, Ukraine; ${ }^{2}$ Alliance Consultancy, Kyiv, Ukraine

\subsection{6/sextrans-2021-sti.285}

Background HIV in Ukraine continues to be concentrated among PWID. According to the IBBS, only 58\% of HIV-positive PWID are aware of their status, so more than 33.000 PWID living with HIV remain undiagnosed. The mapping of PWID locations makes it possible to optimize the HIV casefinding activities, which include both stationary testing sites and outreach routes.

Methods We used community-based participatory research (CBPR) approach to mapping the locations of PWID in 12 cities. The study was conducted in collaboration with leading community-based organizations. We identified the locations in each city, the approximate number of PWID in its, and the schedule for visiting each location (peak days and hours). Data on current locations of HIV testing sites and outreach routes were used for additional analysis.

Results 885 PWID locations of 24 different types were identified (from 31 to 223 in each city). Among them are pharmacies, streets/yards/garages, shops, markets, places of drug sale, parks, places of PWID accumulation, pawnshops/banks, hospitals, transport stops, cafes, entertainment points, railway stations, probation, rehabilitation centers, social and psychological assistance points. $63.3 \%$ of locations are not associated with medical and social services and were previously unknown to HIV-services. Locations were visited all days of the week from $6.00 \mathrm{am}$ to $0.05 \mathrm{am}$.

The mapping results were visualized using Google Maps and QGIS and compared with HIV-projects data. The current location of the sites and outreach routes does not allow the full reach of PWID and relies on the most famous gathering places.

Conclusions Data on the PWID locations are often incompletely known to HIV testing services, which limits the successful HIV cases-finding among them. Experience with community-based mapping has made it possible to re-plan routes and relocate fixed sites to better reach PWID. Obtaining such comprehensive results would not be impossible without collaboration with PWID-community.

\section{P195 SEXUALIZED DRUG USE AMONG FEMALE SEX WORKERS FROM EIGHT CITIES IN CHINA}

${ }^{1} \mathrm{M}$ Xiong*, ${ }^{2} \mathrm{~J}$ Ong, ${ }^{1} \mathrm{Y}$ Wang, ${ }^{1} \mathrm{~W}$ Tang, ${ }^{1} \mathrm{C}$ Wang. ${ }^{1}$ Dermatology Hospital of Southern Medical University, Guangzhou, China; 'Central Clinical School, Monash University, Melbourne, Australia

\subsection{6/sextrans-2021-sti.286}

Background There is rich literature on sexualized drug use (i. e. drug use before or during sex) for men who have sex with men but less data from female sex workers (FSW) particularly from low- and middle-income countries. We describe the sociodemographic characteristics, sexual behaviors and HIV and sexually transmitted infection (STI) testing behaviors among FSW reporting sexualized drug use, compared with FSW who do not report sexualized drug use.

Methods This cross-sectional study was conducted in eight cities in China in 2019. We recruited FSW through community organizations working with sex workers. Multivariable logistic regression models were used to evaluate the factors associated with FSW reporting sexualized drug use. We adjusted for current injecting drug use, age, marital status, migration status, income and education in each of the models.

Results In total, 1287 women participated: average age was $35 \cdot 1$ years (SD 10.3), about half reported a monthly income over 5000 RMB (\$USD 707, 52.4\%), and a minority completed high school or above (17.4\%). Among participants, 284 $(22 \cdot 1 \%$, 95\% CI:19.8-24.4) reported a history of sexualized drug use. Compared to FSW who never reported a history of sexualized drug use or IDU, FSW who reported a history of sexualized drug use had greater odds of: having a manager (adjusted odds ratio (AOR) 2.10, 95\% CI:1.47-2.99), reporting inconsistent condom use for vaginal sex (AOR 2.67, 95\% CI:1.93-3.69), inconsistent condom use for oral sex (AOR 2.33, 95\% CI:1.25-4.37), ever had an unintended pregnancy (AOR 1.43, 95\% CI:1.04-1.96), and ever diagnosed with STIs (AOR 5·39, 95\% CI:3·92-7·40).

Conclusion We recommend routinely asking FSW about sexualized drug use as nearly one in five FSW reported a history of sexualized drug use and these women had an elevated risk profile compared with those who reported no sexualized drug use. 